\title{
Exercise Interventions and Cardiovascular Health in Childhood Cancer: A Meta-analysis
}

\author{
Authors \\ Javier S. Morales ${ }^{1}$, Pedro L. Valenzuela², Alba M. Herrera-Olivares ${ }^{1}$, Antonio Baño-Rodrigo ${ }^{3}$, Adrián Castillo-García ${ }^{4}$, \\ Cecilia Rincón-Castanedo ${ }^{1}$, Asunción Martín-Ruiz ${ }^{1}$, Alejandro F. San-Juan ${ }^{5}$, Carmen Fiuza-Luces ${ }^{6}$, Alejandro Lucia ${ }^{1}$
}

\section{Affiliations}

1 Universidad Europea de Madrid, Faculty of Sport Sciences, Madrid, Spain

2 Department of Systems Biology, University of Alcalá, Madrid, Spain

3 Cardiology Departament, Hospital Infantil Universitario Niño Jesús, Madrid, Spain

4 Fissac - Physiology, Health and Physical Activity, Exercise and Health, Madrid, Spain

5 Laboratorio de Biomecánica Deportiva, Departamento de Salud y Rendimiento Humano, Facultad de Ciencias de la Actividad Física y del Deporte Universidad Politecnica de Madrid, Madrid, Spain

6 Research Institute of the Hospital 12 de Octubre (i+12) Madrid, Spain

Key word

cardiovascular function, exercise is medicine,

cardiorespiratory fitness, physical activity, physical capacity, pediatric cancer

accepted $\quad 20.11 .2019$

\section{Bibliography}

DOI https://doi.org/10.1055/a-1073-8104

Published online: 14.1.2020

Int J Sports Med 2020; 41: 141-153

(c) Georg Thieme Verlag KG Stuttgart · New York

ISSN 0172-4622

Correspondence

Dr. Carmen Fiuza-Luces

12th of October Hospital Research Institute, Physiology,

Madrid

Spain

Tel.: +34 0912115200

cfiuza.imas12@h12o.es

\section{ABSTRACT}

This study analyzed the effects of physical exercise interventions on cardiovascular endpoints in childhood cancer survivors. Relevant articles were systematically searched in PubMed, CINAHL, and Web of Science databases (since inception to 11th September 2019). We performed a meta-analysis (random effects) to determine the mean difference (expressed together with $95 \%$ confidence intervals) between pre- and post-intervention values for those cardiovascular endpoints reported in more than three studies. Twenty-seven studies (of which 16 were controlled studies) comprising 697 participants were included. Only three studies reported adverse events related to exercise interventions. Exercise resulted in an increased performance on the 6-minute walk distance test (mean difference $=111 \mathrm{~m}, 95 \%$ confidence interval $=39-183, \mathrm{p}=0.003$ ) and a non-significant trend (mean difference $=1.97 \mathrm{ml} \cdot \mathrm{kg}^{-1} \cdot \mathrm{min}^{-1}$, $95 \%$ confidence interval $=-0.12-4.06, p=0.065)$ for improvement in peak oxygen uptake. Furthermore, left ventricular ejection fraction was preserved after exercise interventions (mean difference $=0.29 \%, 95 \%$ confidence interval $=-1.41-1.99$, $\mathrm{p}=0.738$ ). In summary, exercise interventions might exert a cardioprotective effect in childhood cancer survivors by improving - or attenuating the decline of - physical capacity and cardiovascular function. Further studies, particularly randomized controlled trials, are needed to confirm these benefits.

\section{Introduction}

Despite treatment advances and improvements in 5-year survival rates, pediatric cancer survivors frequently experience adverse events related to the disease and its treatment [1]. Cardiovascular disease is the main cause of morbidity and mortality among child- hood cancer survivors (CCS) [2, 3]. Anthracycline-induced cardiotoxicity is among the most frequent adverse effects observed in this patient population [4,5], although CCS present with a lower left ventricular function (i. e., echocardiography-assessed ejection [LVEF] and fractional shortening [FS]) than their healthy peers irre- 
spective of the treatment received - that is, whether or not they had been treated with anthracyclines [6]. Thus, developing effective cardioprotective strategies is a clinical aspiration.

Dexrazoxane (ICRF-187) is arguably the most studied of the available cardioprotective agents and is associated with a statistically significant risk reduction for most cardiotoxic outcomes [7]. However, evidence suggests that in some contexts (e. g., when supplied concurrently with cancer therapies) this medication may increase the risk of developing second malignant neoplasms [7]. Exercise interventions have proven to be safe and to play a cardioprotective role by improving or at least mitigating cancer/treatment-related adverse effects on cardiovascular status. For instance, we recently found that in-hospital exercise interventions in CCS during treatment attenuated the impairment in LV function without increasing the risk of mortality, disease relapse, or metastasis [8]. Furthermore, there is meta-analytic evidence supporting the effectiveness of physical exercise for the improvement of cardiorespiratory fitness (CRF) in this population [9]. However, to our knowledge there is no meta-analytical evidence supporting the effectiveness of physical exercise interventions on the cardiovascular system in this patient population. Accordingly, in the present systematic review and meta-analysis, we aimed to summarize the evidence on the effects of exercise training intervention (duration $\geq 3$ weeks) on cardiovascular endpoints in CCS.

\section{Materials and Methods}

The conduct and reporting of the current systematic review and meta-analysis conform to the Preferred Reporting Items for Systematic Reviews and Meta-analyses [10] as well as journal standards [11].

\section{Systematic search}

Two authors (J.S.M. and P.L.V.) independently performed the systematic search for relevant articles written in English and screened by title and abstract in the electronic databases PubMed, CINAHL, and Web of Science (from inception to September 11, 2019) using the following strategy: (child * OR adolescen * OR pediatric) AND (exercise OR "physical activity" OR training) AND (cancer OR tumor OR neoplasm OR maligna * OR leukemia OR leukaemia OR oncology). The electronic search was supplemented with a manual review of reference lists from relevant publications to locate additional publications. Gray literature (e. g., abstracts, conference proceedings and editorials) and reviews were excluded. Disagreements were resolved through discussion with a third author (A.M.H.O.).

\section{Study selection and data extraction}

Studies were eligible for inclusion if they met all following criteria: a) assessing CCS during or after treatment, b) including an exercise intervention of $\geq 3$ weeks composed of aerobic and/or strength exercises, and c) evaluating $\geq 1$ endpoint related to cardiovascular health. Having a control group (i. e., a group performing no exercise) was not required for a study to be included. In those studies that included a control group of participants who were not CCS, only the results of the intervention group were considered in the analyses. Case studies (i. e., with one or two subjects) were excluded from the analysis.
Two authors (J.S.M. and P.L.V.) independently extracted the following data from each study, if available: number, sex, and age of participants; main cancer characteristics (cancer type, age at diagnosis, time since diagnosis and treatment, time of remission, and anti-cancer treatment received); interventions' characteristics; cardiovascular endpoints assessed; measurement methods; and main results. Main outcomes' data were extracted as mean and standard deviation (SD) or in a manner allowing their transformation into mean and SD. Two studies provided the median and interquartile range instead of the mean and SD $[12,13]$ and transformations were made with the formula proposed by Hozo et al [14]. Disagreements were resolved through discussion with a third reviewer (A.C.G.). We contacted the corresponding author of each study when necessary to clarify any uncertainty or to request additional data. In this regard, three authors provided the required specific data upon request: San Juan et al. [15], Long et al. [16], and Rath et al. [13].

\section{Quality assessment of the included studies}

The methodological quality of the included studies was assessed with the PEDro scale, which is based on the Delphi list [17]. Two authors (J.S.M and A.C.G.) independently scored the studies, and disagreements were resolved through discussion with a third author (A.M.H.O.). A total score of $0-10$ was determined by counting the number of criteria satisfied by each study (see footnote in $>$ Table 1 for a brief description of criteria). Study quality was rated as poor (PEDro score $\leq 3)$, fair (4-5), or high (>5).

\section{Statistical analysis}

We performed a meta-analysis using a random effects model to compute the mean difference (MD, expressed along with the $95 \%$ confidence interval $[\mathrm{Cl}]$ ) between pre- and post-intervention data for those endpoints assessed in $\geq 3$ studies and using the same assessment method. When possible, we performed a sub-analysis based on whether participants were still under treatment or not. The weight assigned to each study in the meta-analysis was defined by the SD of the endpoints and the sample size. Begg's test was used to determine the presence of publication bias, and the $Q$ and $I^{2}$ statistics were used to assess heterogeneity across studies. The level of significance was set at 0.05 . All statistical analyses were performed using the statistical software package MIX 2.0 Pro for Excel software [18].

\section{Results}

\section{Study selection}

From the retrieved articles, 27 studies $[8,12,13,15,16,19-40]$ were finally included in the systematic review ( $\triangleright$ Fig. 1). The characteristics of the included studies are summarized in $>$ Tables 2 and $\mathbf{} \mathbf{3}$. Sixteen studies included a non-exercising control group $[8,12,15,16,19-21,23,25,27,31,34-36,38,39]$, of which seven were randomized controlled trials [20, 21, 25, 27, 31, 34, 36], five used a parallel design $[8,12,15,19,35]$, three used a crossover design $[16,38,39]$, two used a control group composed of participants who were not CCS $[15,19]$, and one used a 'historical' control group whose medical conditions were comparable with those 
- Table 1 Quality of the studies included in the systematic review.

\begin{tabular}{|c|c|c|c|c|c|c|c|c|c|c|c|c|}
\hline \multicolumn{13}{|l|}{ Items } \\
\hline Study & 1 & 2 & 3 & 4 & 5 & 6 & 7 & 8 & 9 & 10 & 11 & $\begin{array}{l}\text { Total } \\
\text { Score }\end{array}$ \\
\hline Beulertz et al. [19] & + & - & $?$ & - & $?$ & $?$ & - & + & $?$ & + & + & 3 \\
\hline Braam et al. [20] & + & + & + & + & $?$ & $?$ & + & + & + & + & + & 8 \\
\hline Braam et al. [21] & + & + & + & + & $?$ & $?$ & + & + & + & + & + & 8 \\
\hline Chamorro-Viña et al. [23] & + & $\mathrm{N} / \mathrm{A}$ & $\mathrm{N} / \mathrm{A}$ & + & $\mathrm{N} / \mathrm{A}$ & $\mathrm{N} / \mathrm{A}$ & + & + & $?$ & + & + & 5 \\
\hline Cox et al. [25] & + & + & + & + & $?$ & + & $?$ & + & $?$ & + & + & 7 \\
\hline Dubnov-Raz et al. [12] & + & - & $\mathrm{N} / \mathrm{A}$ & + & - & - & - & + & $?$ & + & + & 4 \\
\hline Esbenshade et al. [26] & + & $\mathrm{N} / \mathrm{A}$ & $\mathrm{N} / \mathrm{A}$ & $\mathrm{N} / \mathrm{A}$ & $\mathrm{N} / \mathrm{A}$ & $\mathrm{N} / \mathrm{A}$ & $\mathrm{N} / \mathrm{A}$ & - & $?$ & $\mathrm{~N} / \mathrm{A}$ & - & 0 \\
\hline Fiuza-Luces et al. [27] & + & + & + & + & $?$ & $?$ & + & + & + & + & + & 8 \\
\hline Järvelä et al. [28] & + & $\mathrm{N} / \mathrm{A}$ & $\mathrm{N} / \mathrm{A}$ & $\mathrm{N} / \mathrm{A}$ & $\mathrm{N} / \mathrm{A}$ & $\mathrm{N} / \mathrm{A}$ & $\mathrm{N} / \mathrm{A}$ & + & $?$ & $\mathrm{~N} / \mathrm{A}$ & + & 2 \\
\hline Järvelä et al. [29] & + & $\mathrm{N} / \mathrm{A}$ & $\mathrm{N} / \mathrm{A}$ & $\mathrm{N} / \mathrm{A}$ & $\mathrm{N} / \mathrm{A}$ & $\mathrm{N} / \mathrm{A}$ & $\mathrm{N} / \mathrm{A}$ & + & $?$ & $\mathrm{~N} / \mathrm{A}$ & + & 2 \\
\hline Järvelä et al. [30] & + & $\mathrm{N} / \mathrm{A}$ & $\mathrm{N} / \mathrm{A}$ & $\mathrm{N} / \mathrm{A}$ & $\mathrm{N} / \mathrm{A}$ & $\mathrm{N} / \mathrm{A}$ & $\mathrm{N} / \mathrm{A}$ & + & $?$ & $\mathrm{~N} / \mathrm{A}$ & + & 2 \\
\hline Keats \& Culos-Reed et al. [32] & + & $\mathrm{N} / \mathrm{A}$ & $\mathrm{N} / \mathrm{A}$ & $\mathrm{N} / \mathrm{A}$ & $\mathrm{N} / \mathrm{A}$ & $\mathrm{N} / \mathrm{A}$ & $\mathrm{N} / \mathrm{A}$ & + & $?$ & $\mathrm{~N} / \mathrm{A}$ & + & 2 \\
\hline Kim \& Park [33] & + & $\mathrm{N} / \mathrm{A}$ & $\mathrm{N} / \mathrm{A}$ & $\mathrm{N} / \mathrm{A}$ & $\mathrm{N} / \mathrm{A}$ & $\mathrm{N} / \mathrm{A}$ & $\mathrm{N} / \mathrm{A}$ & + & $?$ & $\mathrm{~N} / \mathrm{A}$ & + & 2 \\
\hline Long et al. [16] & + & - & $?$ & - & - & - & - & + & $?$ & + & + & 3 \\
\hline Marchese et al. [31] & + & + & $?$ & + & $?$ & $?$ & $?$ & + & $?$ & + & + & 6 \\
\hline Morales et al. [8] & + & - & - & + & - & - & - & + & + & + & + & 5 \\
\hline Moyer-Mileur et al. [34] & + & + & $?$ & + & $?$ & $?$ & $?$ & + & $?$ & + & + & 5 \\
\hline Piscione et al. [38] & + & + & + & - & - & - & - & + & $?$ & + & + & 5 \\
\hline Rath et al. [13] & + & - & $?$ & $\mathrm{~N} / \mathrm{A}$ & - & - & - & - & $?$ & $\mathrm{~N} / \mathrm{A}$ & + & 1 \\
\hline Riggs et al. [39] & + & + & + & - & - & - & - & + & $?$ & + & + & 5 \\
\hline San Juan et al. [24] & + & $\mathrm{N} / \mathrm{A}$ & $\mathrm{N} / \mathrm{A}$ & $\mathrm{N} / \mathrm{A}$ & $\mathrm{N} / \mathrm{A}$ & $\mathrm{N} / \mathrm{A}$ & $\mathrm{N} / \mathrm{A}$ & + & $?$ & $\mathrm{~N} / \mathrm{A}$ & + & 2 \\
\hline San Juan et al. [15] & + & $\mathrm{N} / \mathrm{A}$ & $\mathrm{N} / \mathrm{A}$ & $\mathrm{N} / \mathrm{A}$ & $\mathrm{N} / \mathrm{A}$ & $\mathrm{N} / \mathrm{A}$ & $\mathrm{N} / \mathrm{A}$ & + & $?$ & + & + & 3 \\
\hline Shore \& Shepard [35] & + & $\mathrm{N} / \mathrm{A}$ & $\mathrm{N} / \mathrm{A}$ & $\mathrm{N} / \mathrm{A}$ & $\mathrm{N} / \mathrm{A}$ & $\mathrm{N} / \mathrm{A}$ & $\mathrm{N} / \mathrm{A}$ & + & $?$ & + & + & 3 \\
\hline Smith et al. [40] & + & $\mathrm{N} / \mathrm{A}$ & $\mathrm{N} / \mathrm{A}$ & $\mathrm{N} / \mathrm{A}$ & $\mathrm{N} / \mathrm{A}$ & $\mathrm{N} / \mathrm{A}$ & $\mathrm{N} / \mathrm{A}$ & + & $?$ & $\mathrm{~N} / \mathrm{A}$ & - & 1 \\
\hline Su et al. [37] & + & $\mathrm{N} / \mathrm{A}$ & $\mathrm{N} / \mathrm{A}$ & $\mathrm{N} / \mathrm{A}$ & $\mathrm{N} / \mathrm{A}$ & $\mathrm{N} / \mathrm{A}$ & $\mathrm{N} / \mathrm{A}$ & - & + & $\mathrm{N} / \mathrm{A}$ & + & 2 \\
\hline Takken et al. [22] & + & $\mathrm{N} / \mathrm{A}$ & $\mathrm{N} / \mathrm{A}$ & $\mathrm{N} / \mathrm{A}$ & $\mathrm{N} / \mathrm{A}$ & $\mathrm{N} / \mathrm{A}$ & $\mathrm{N} / \mathrm{A}$ & - & $?$ & $\mathrm{~N} / \mathrm{A}$ & - & 0 \\
\hline Tanir \& Kuguoglu [36] & + & + & $?$ & + & $?$ & $?$ & $?$ & + & $?$ & + & + & 5 \\
\hline \multicolumn{13}{|c|}{$\begin{array}{l}\text { Column numbers correspond to the following criteria on the PEDro scale: } 1 \text { - Eligibility criteria were specified } 2 \text { - Subjects were randomly allocated to } \\
\text { groups (or, in a crossover study, subjects were randomly allocated an order in which treatments were received) } 3 \text { - Allocation was concealed } 4 \text { - Groups } \\
\text { were similar at baseline } 5 \text { - Subjects were blinded } 6 \text { - Therapists who administered the treatment were blinded } 7 \text { - Assessors were blinded } 8 \text { - Measures } \\
\text { of key outcomes were obtained from more than } 85 \% \text { of subjects } 9 \text { - Data were analyzed by intention to treat } 10 \text { - Statistical comparisons between } \\
\text { groups were conducted } 11 \text { - Point measures and measures of variability were provided *A total score out of } 10 \text { is determined from the number of criteria } \\
\text { that are satisfied, except that scale item } 1 \text { is not used to generate the total score. + Indicates the criterion was clearly satisfied; - indicates that it was not; ? } \\
\text { indicates that it is not clear whether the criterion was satisfied; N/A indicates that it was not applicable. }\end{array}$} \\
\hline
\end{tabular}

of the intervention group [23]. The remaining studies were noncontrolled trials $[13,22,24,26,28-30,32,33,37,40]$.

\section{Quality assessment and publication bias}

The quality of the included studies was overall fair (median PEDro score $=4$, range $0-8 ; \triangleright$ Table 1 ). Fifteen studies were deemed to have poor methodological quality $[13,15,16,19,22,24,26,28-$ $30,32,33,35,37,40]$, seven had fair quality $[8,12,23,34,36,38,39]$, and the remainder were considered to present a high quality $[20,21,25,27,31]$.

\section{Characteristics of participants and interventions}

The retrieved studies included a total of 697 participants (range 4-169), of which 669 were CCS and 28 were healthy children. Two participants from two studies were excluded because they had noncancer-related hematological disorders [12,33]. Some studies ana- lyzed the same sample (Braam et al. [20,21], Järvela et al. [28-30], Riggs et al. [39], and Piscione et al. [38]), and thus we used only one study in each case to compute the total number of subjects.

The included studies analyzed children/adolescents and adult survivors (average age range 5-38 years) with different types of childhood cancer (the most common being leukemias) during or after treatment completion. Sixteen studies [8, 15, 19-21, 23$27,31,32,34-37]$ analyzed the effects of physical exercise interventions in CCS during treatment (or at least $50 \%$ of the included CCS were in active treatment) and 11 studies $[12,13,16,22,28-$ $30,33,38-40$ ] analyzed CCS who had already finished anti-cancer therapy. The age at diagnosis ranged from zero to 15 years, the time since diagnosis from one to 22 years, and the time since the end of treatment of those CCS who had already finished treatment from zero to 21 years. Three of the included studies did not report 


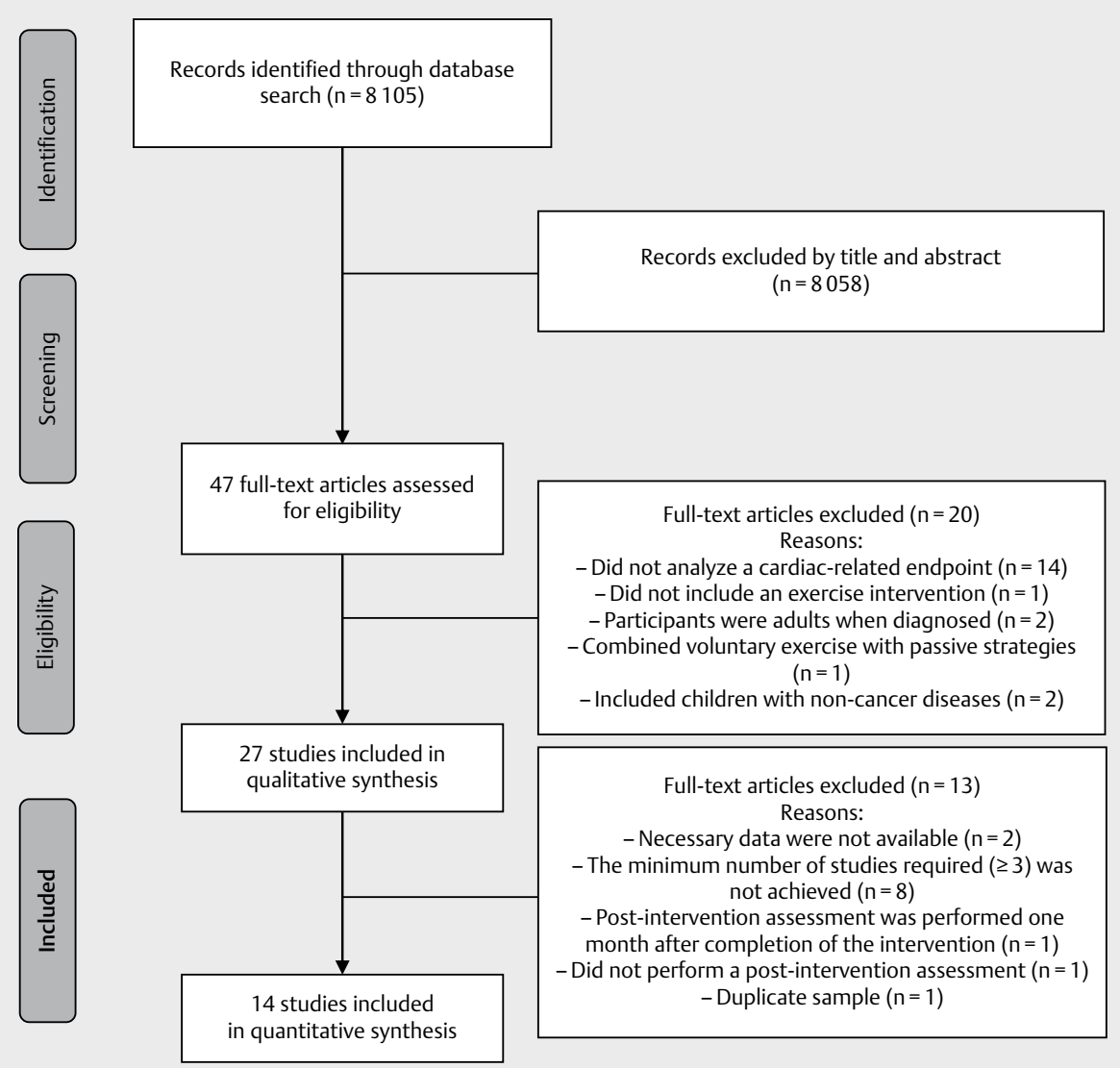

- Fig. 1 Flow chart of literature search.

the age at diagnosis, four did not report the time since diagnosis, and four did not report the time since the end of treatment.

Exercise interventions were supervised in 16 studies $[8,12,13$, $15,16,19-24,27,32,33,38,39]$ and mixed in four [ $25,31,35,37]$. The remainder were not supervised $[26,28-30,34,36,40]$. Exercise interventions consisted of aerobic exercise [35, 37-39] or a combination of both aerobic and resistance exercise $[8,12,13,15,16,19-$ $34,36,40$ ], lasted 3 weeks to 2.5 years, and were performed 1-6 days per week. Exercise intensity ranged from 50 to $60 \%$ of onerepetition maximum for resistance exercise, and between 50 and $>90 \%$ heart rate peak for aerobic exercise. Fourteen out of the 27 studies did not register exercise intensity.

The prevalence of adverse events associated with the exercise intervention was reported in 16 studies [8, 13, 15, 19-24, 27, 31 $33,37,39,40]$, and only three of them found adverse events $[13,19,22]$ : in the study of Beulertz et al. [19] a participant dislocated his patella, Rath et al. [13] reported a fall during an exercise session, and Takken et al. [22] reported headache, muscle soreness, fatigue, and hyperventilation during the exercise interventions.

\section{Endpoints}

\section{Cardiorespiratory fitness}

Twelve studies [12, 15, 16, 20-22, 24, 27, 28, 35, 38, 40] analyzed CRF through the measurement of peak oxygen uptake $\left(\mathrm{VO}_{2 \text { peak }}\right)$. Of these, nine could be meta-analyzed, showing a non-significant trend towards an improvement in $\mathrm{VO}_{2 \text { peak }}\left(\mathrm{n}=118, \mathrm{MD}=1.97 \mathrm{ml} \cdot \mathrm{kg}^{-1} \cdot \mathrm{min}^{-1}\right.$, $95 \% \mathrm{Cl}=0.12-4.06, p=0.065$, $\boldsymbol{\text { Fig. }}$. $2 \mathrm{a}$ ), with no signs of heterogeneity $\left(Q=4.633, \mathrm{I}^{2}=0 \%\right)$ and no signs of publication bias $(\mathrm{p}=0.602)$. Three studies $[20,35,38]$ could not be meta-analyzed because postintervention assessment was performed one month after completion of the exercise intervention [20], data could not be obtained because the authors could not be contacted [35], and the authors could not analyze the data because of participants' refusal and difficulties performing the necessary procedures [38]. In the latter case, Piscione et al. [38] used pro-rated work rate as a proxy measure of CRF, finding a significant improvement after the exercise intervention. Sub-analyses revealed no differences for $\mathrm{VO}_{2 \text { peak }}$ in CCS who were still under treatment $\left(\mathrm{n}=69, \mathrm{MD}=2.26 \mathrm{ml} \cdot \mathrm{kg}^{-1} \cdot \mathrm{min}^{-1}, 95 \% \mathrm{Cl}=-0.54-5.07\right.$, $p=0.114)$ or who had already finished anti-cancer therapy $(n=49$, $\left.\mathrm{MD}=1.62 \mathrm{ml} \cdot \mathrm{kg}^{-1} \cdot \mathrm{min}^{-1}, 95 \% \mathrm{Cl}=-2.62-5.86, p=0.453\right)$.

Ten studies $[19,25,26,31-34,36,37,39]$ analyzed the effects of exercise on CRF assessed with submaximal tests. Four studies $[25,26,37,39]$ evaluated CRF using the 6 -minute walk distance (6MWD) test, although data from one [39] study could not be obtained despite contacting the authors. From the three studies meta-analyzed, a significant increase in 6MWD performance was found ( $\mathrm{n}=66, \mathrm{MD}=111 \mathrm{~m}, 95 \% \mathrm{Cl}=39-183, p=0.003$, $>$ Fig. 2 b) with no evidence of publication bias $(p=1)$ but with heterogeneity between 


\begin{tabular}{|c|c|c|c|c|c|c|}
\hline & 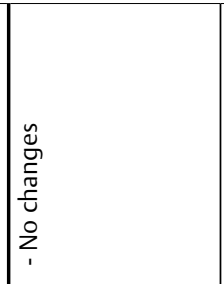 & 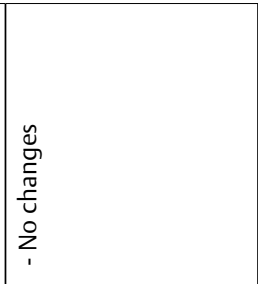 & 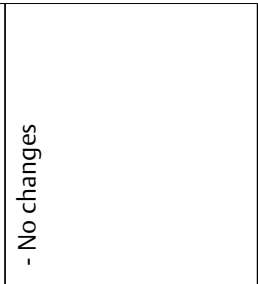 & 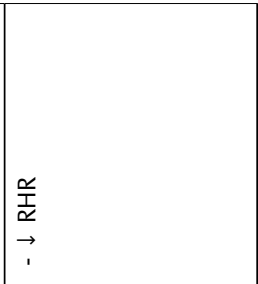 & 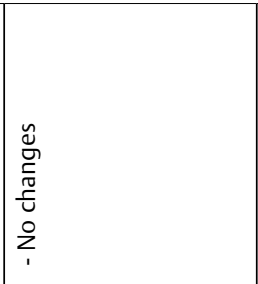 & 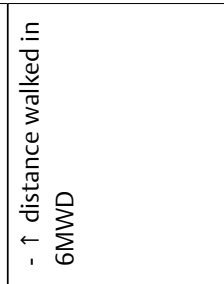 \\
\hline 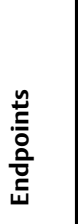 & 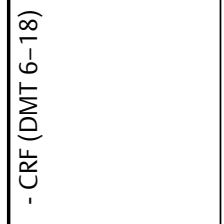 & 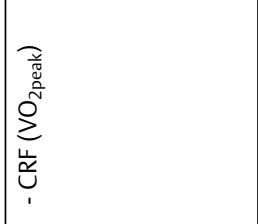 & 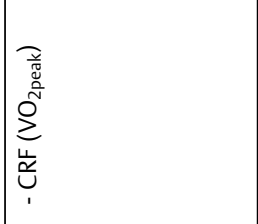 & 垃 & $\mid$ & 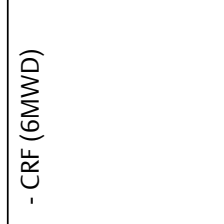 \\
\hline 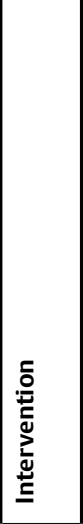 & 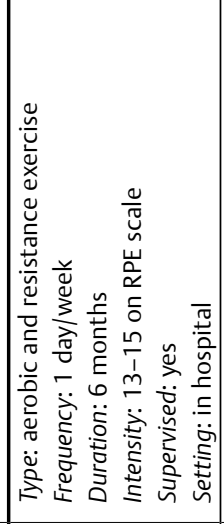 & 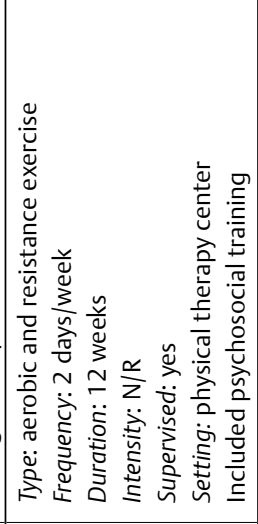 & 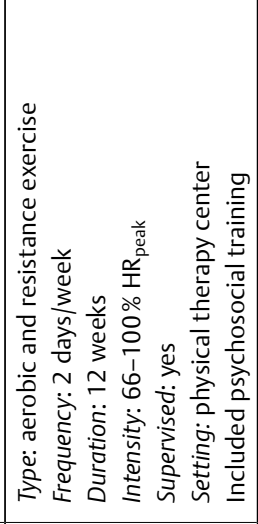 & 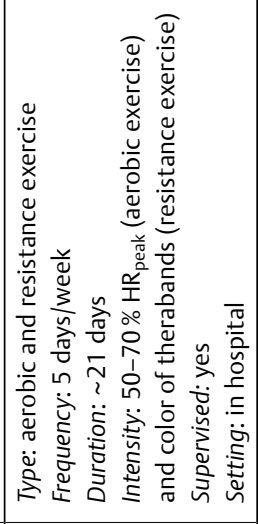 & 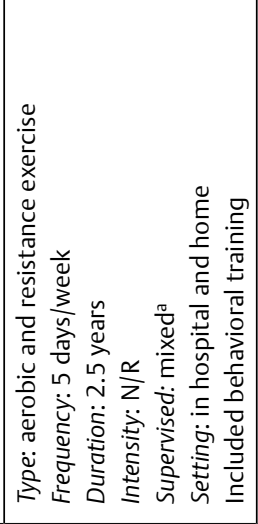 & 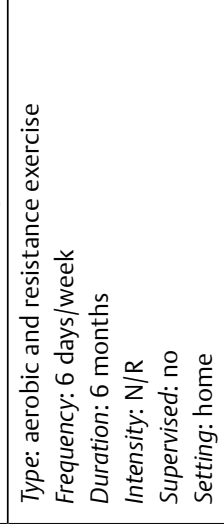 \\
\hline 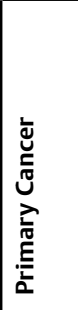 & 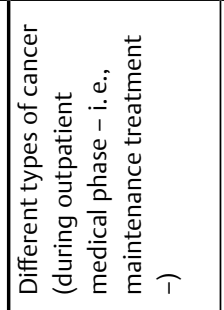 & 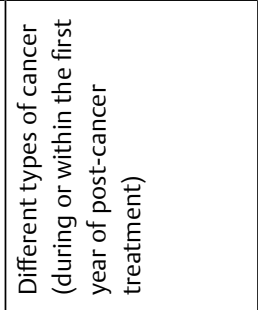 & 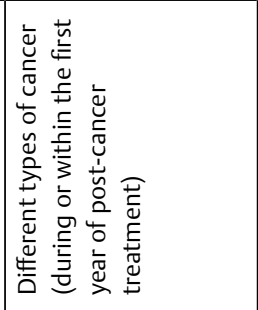 & 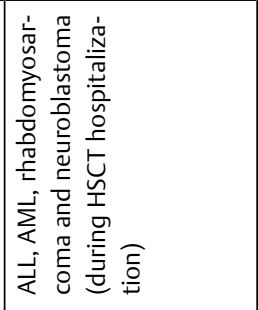 & 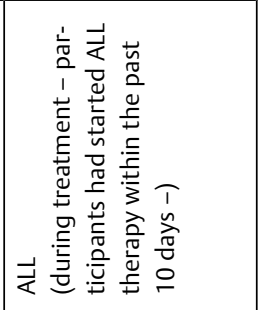 & 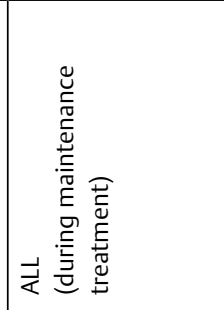 \\
\hline 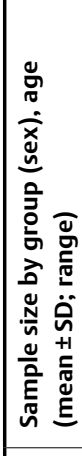 & 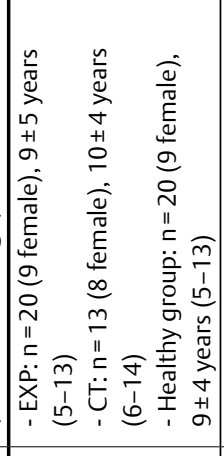 & 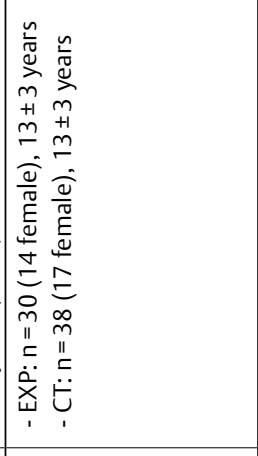 & 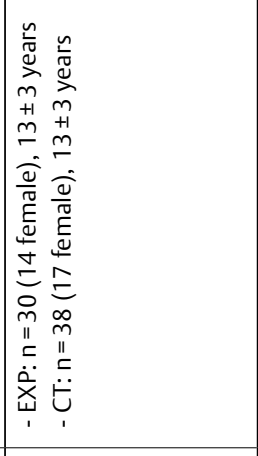 & 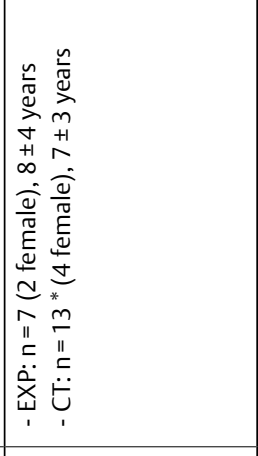 & 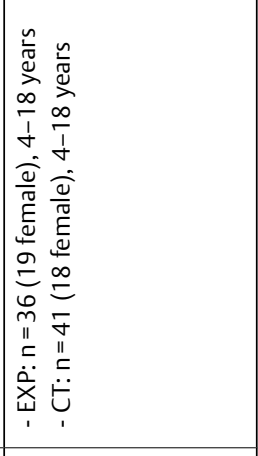 & 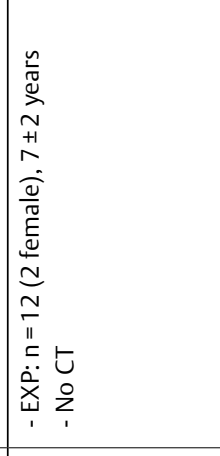 \\
\hline 童 & 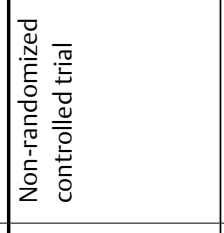 & 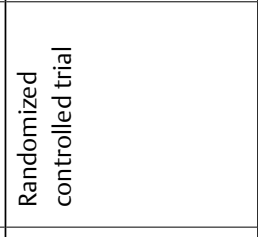 & 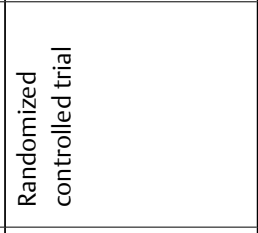 & 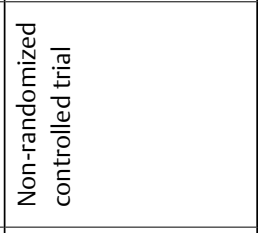 & 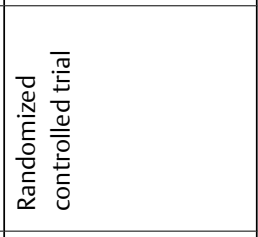 & 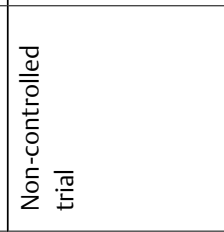 \\
\hline 㐔 & 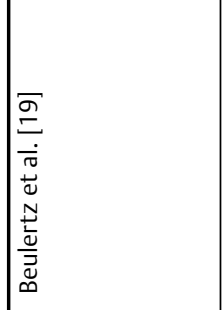 & 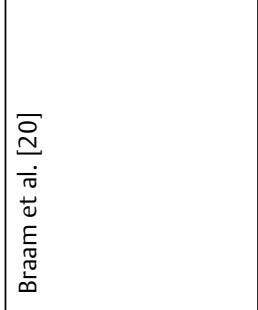 & 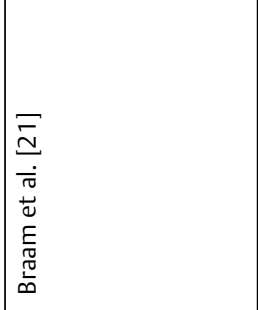 & 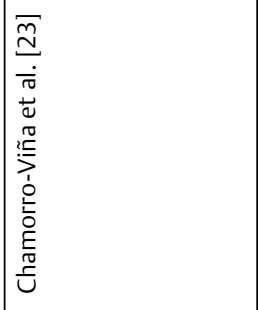 & 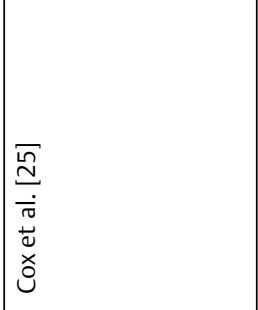 & 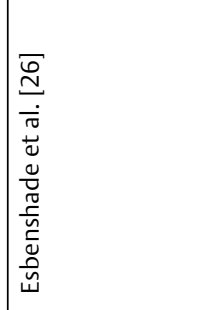 \\
\hline
\end{tabular}




\begin{tabular}{|c|c|c|c|c|c|c|}
\hline 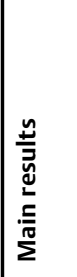 & 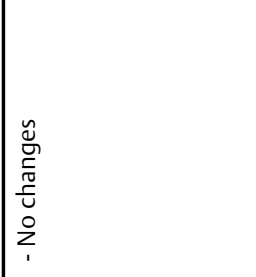 & 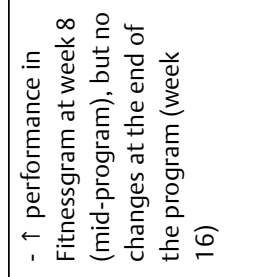 & 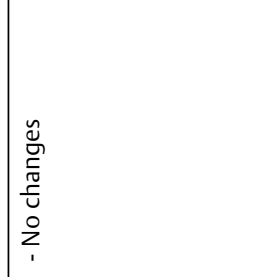 & 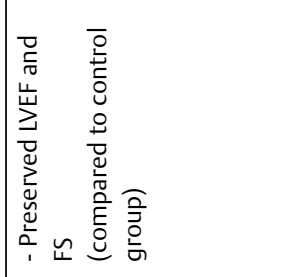 & 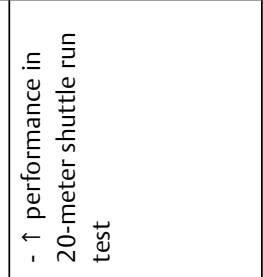 & 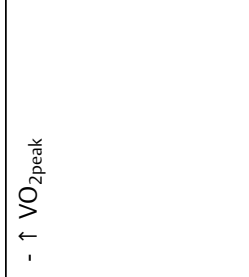 \\
\hline 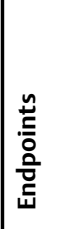 & 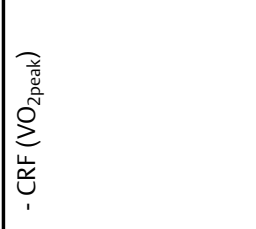 & 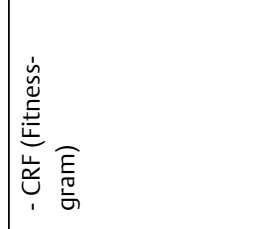 & 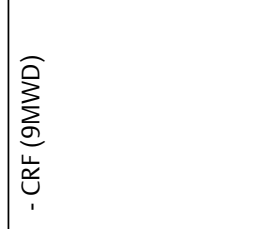 & 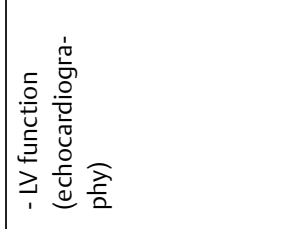 & 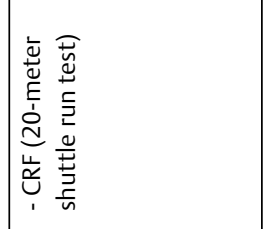 & 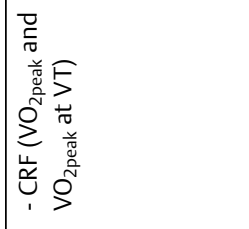 \\
\hline 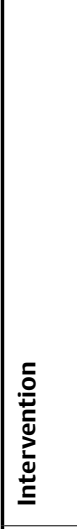 & 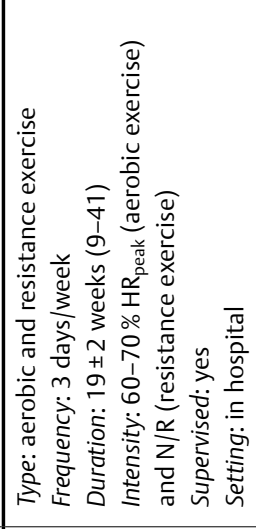 & 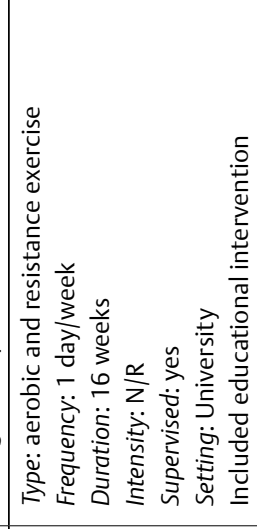 & 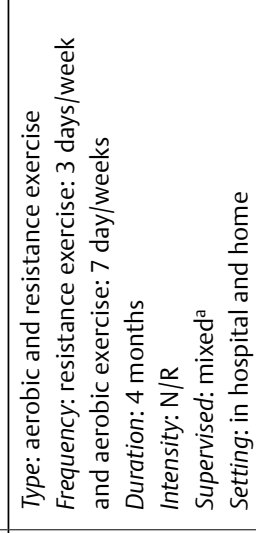 & 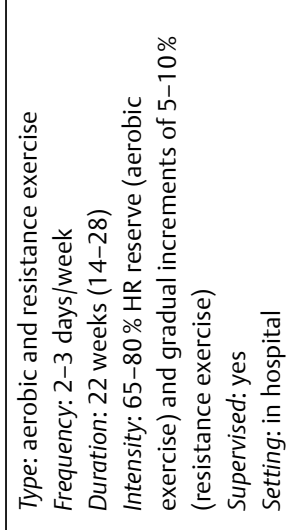 & 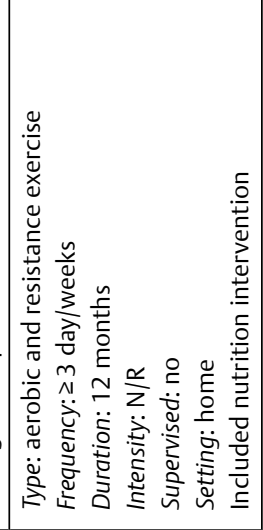 & 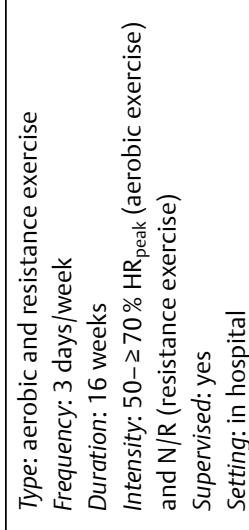 \\
\hline 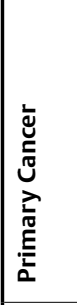 & 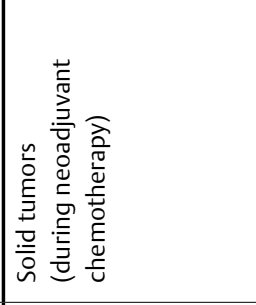 & 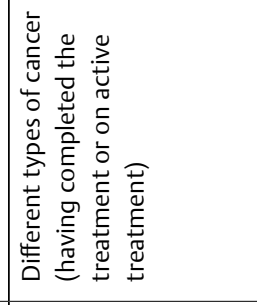 & 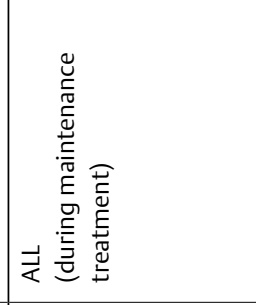 & 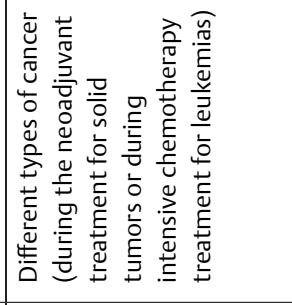 & 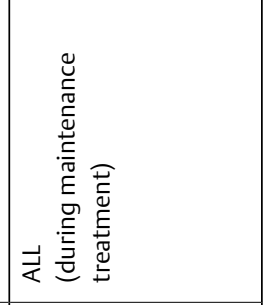 & 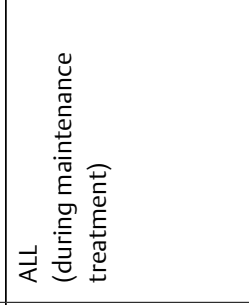 \\
\hline 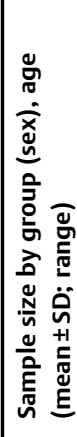 & 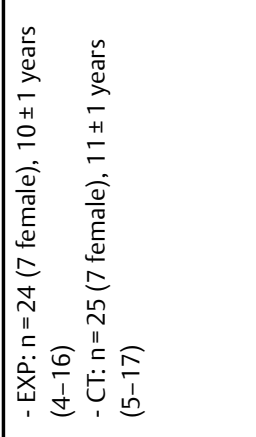 & 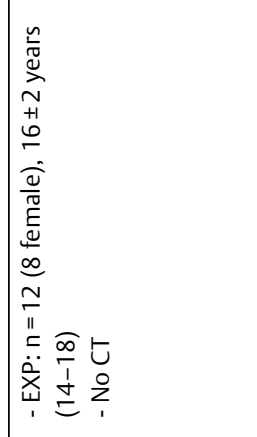 & 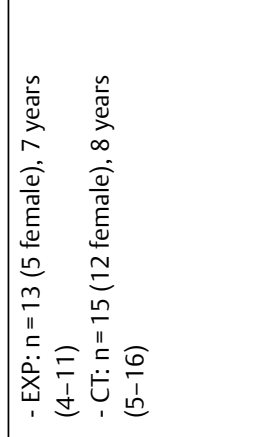 & 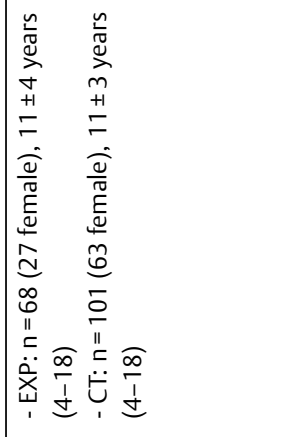 & 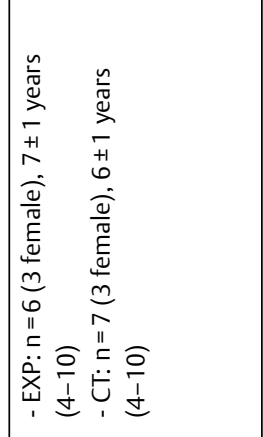 & 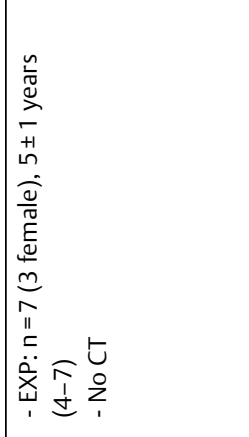 \\
\hline 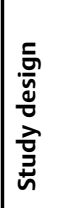 & 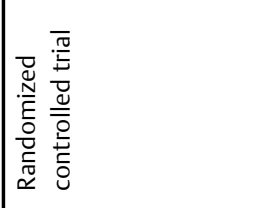 & 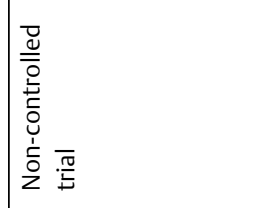 & 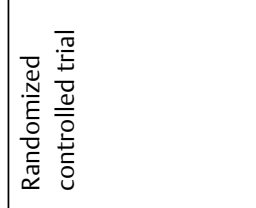 & 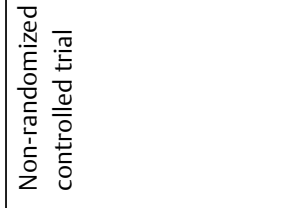 & 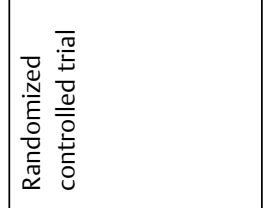 & 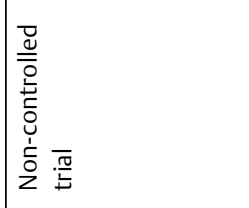 \\
\hline 호 & 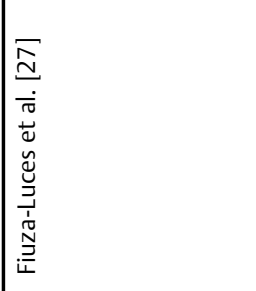 & 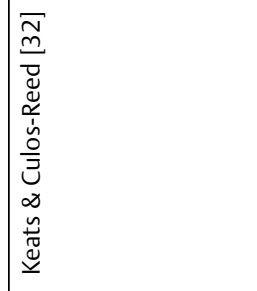 & 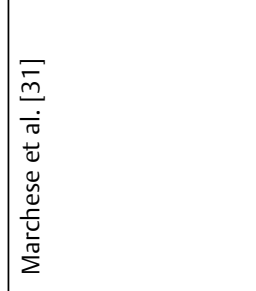 & 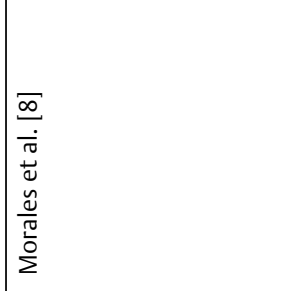 & 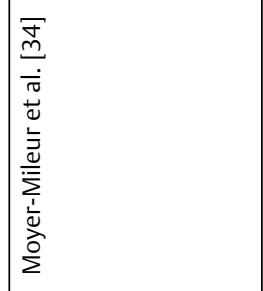 & 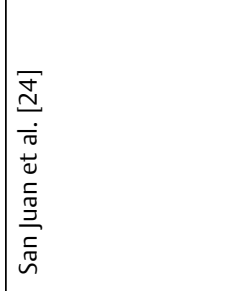 \\
\hline
\end{tabular}




\begin{tabular}{|c|c|c|c|c|c|}
\hline & 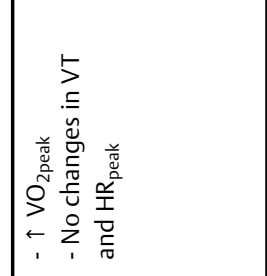 & 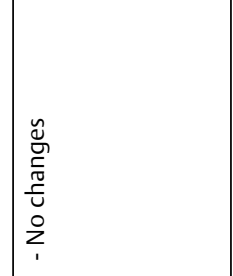 & 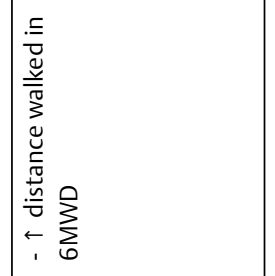 & 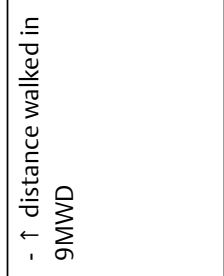 & 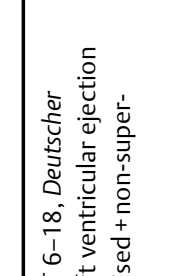 \\
\hline & 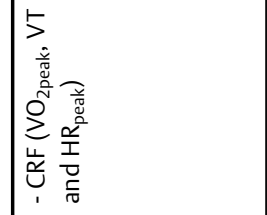 & 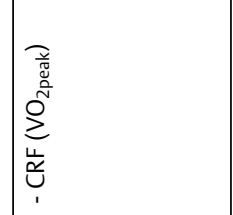 & 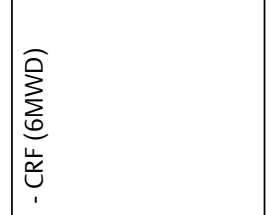 & 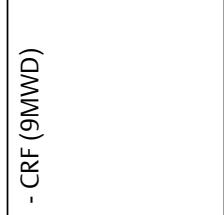 & 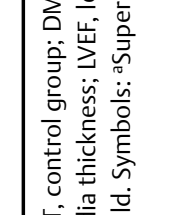 \\
\hline & 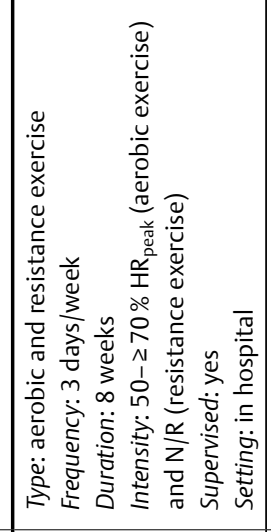 & 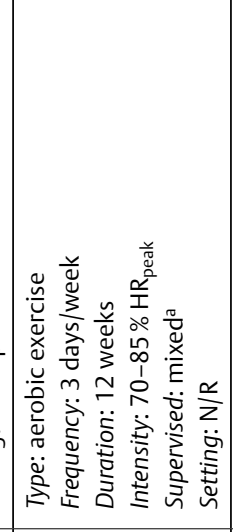 & 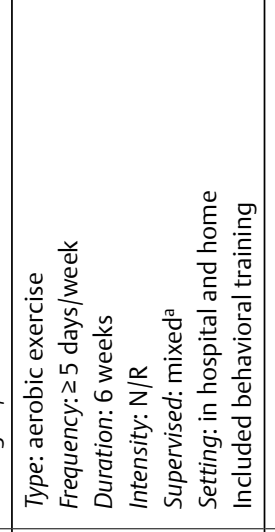 & 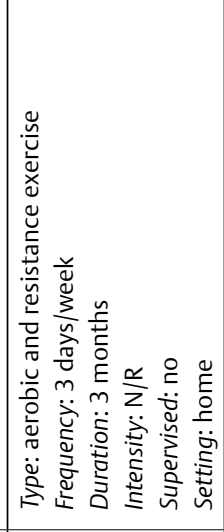 & 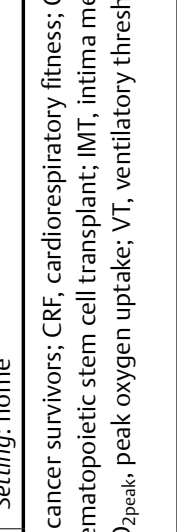 \\
\hline & 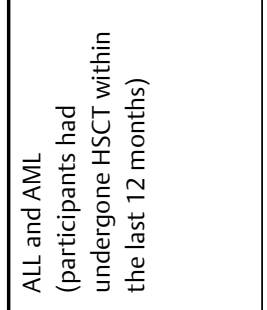 & 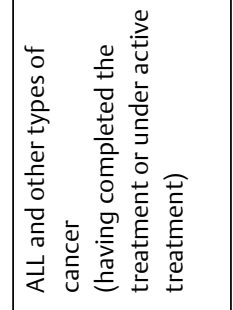 & 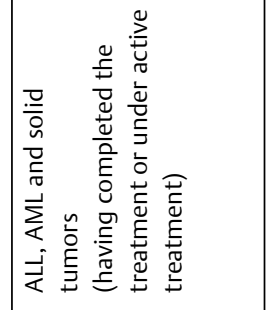 & 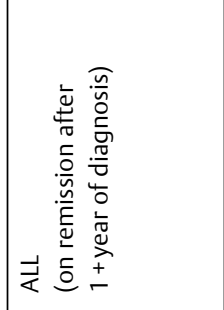 & 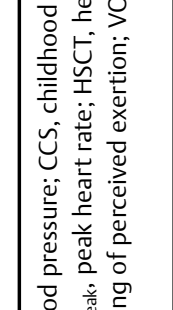 \\
\hline & 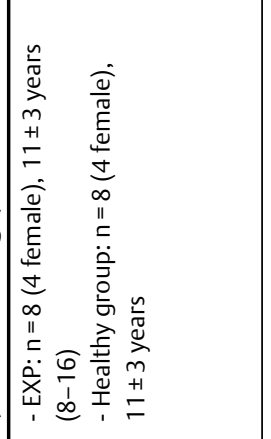 & 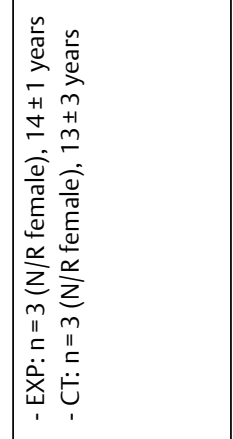 & 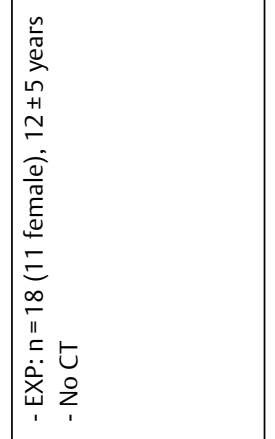 & 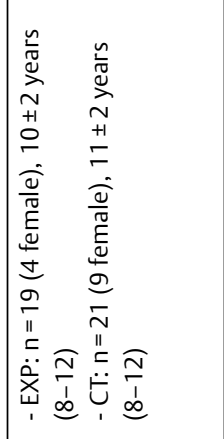 & 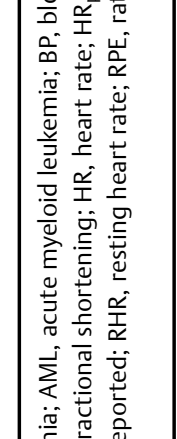 \\
\hline & 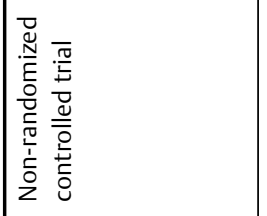 & 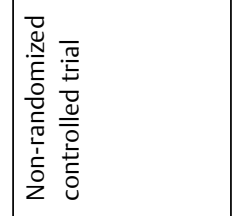 & 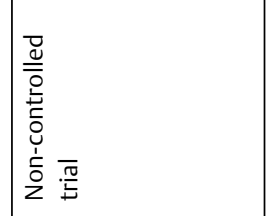 & 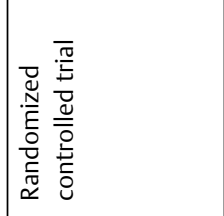 & 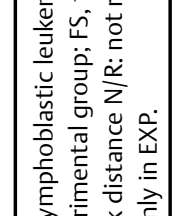 \\
\hline & 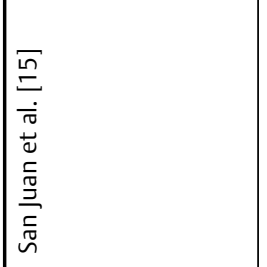 & 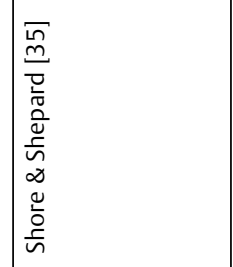 & 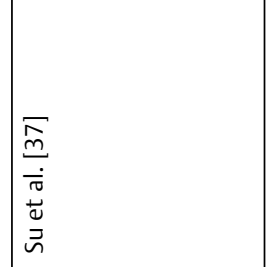 & 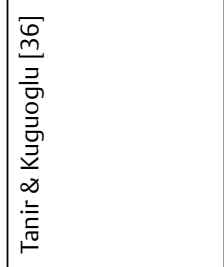 & 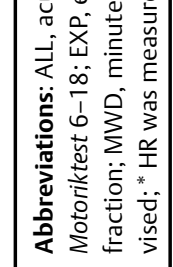 \\
\hline
\end{tabular}




\begin{tabular}{|c|c|c|c|c|c|c|}
\hline 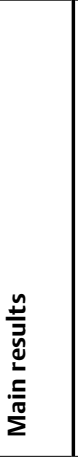 & 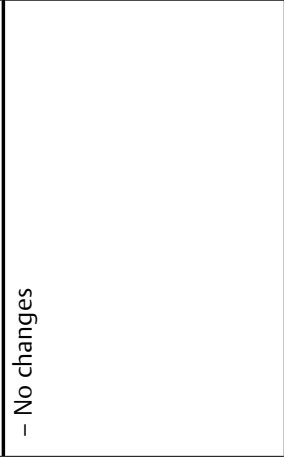 & 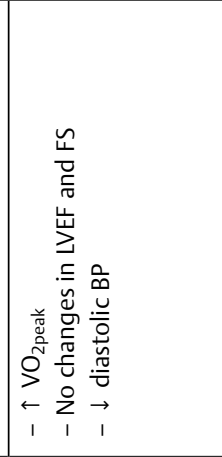 & 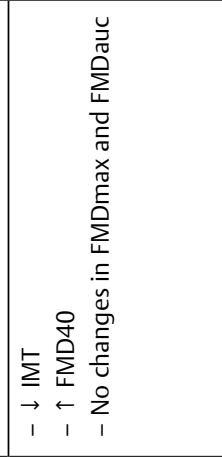 & 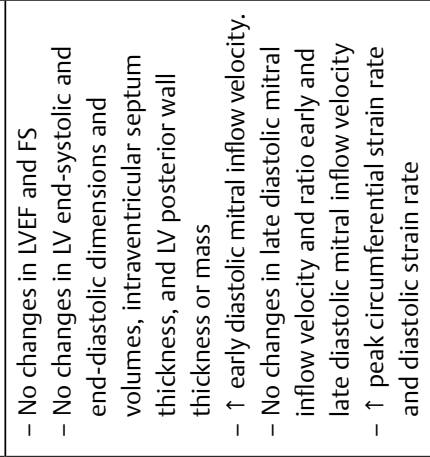 & 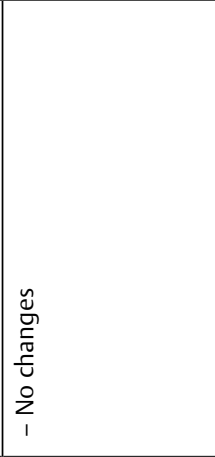 & 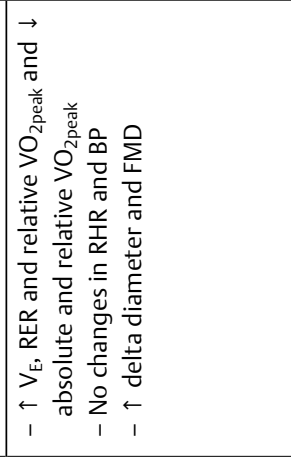 \\
\hline & 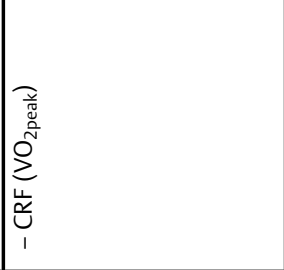 & 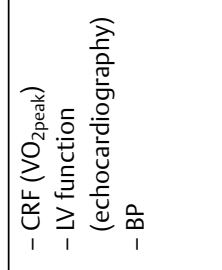 & 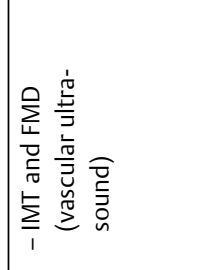 & 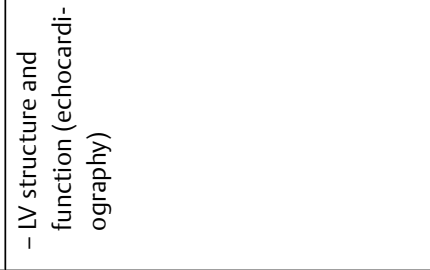 & 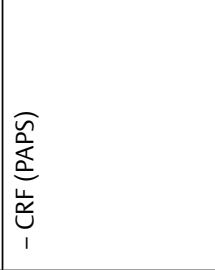 & 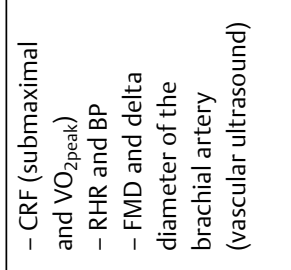 \\
\hline & 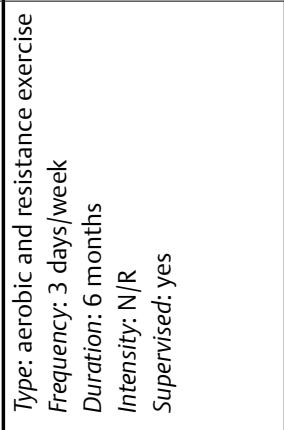 & 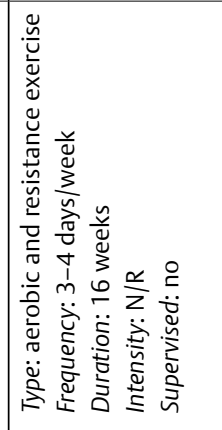 & 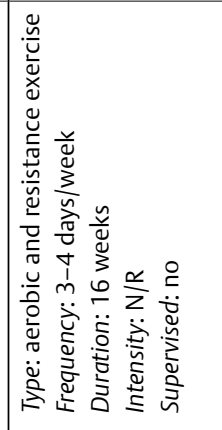 & 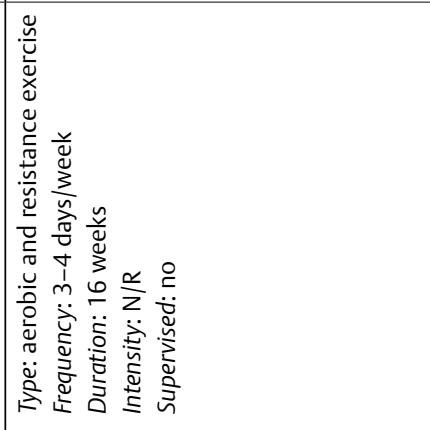 & 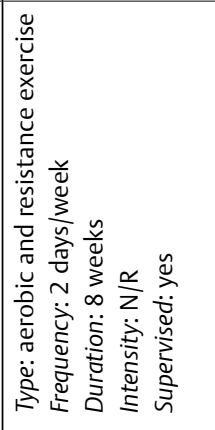 & 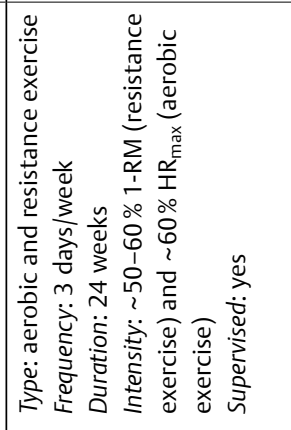 \\
\hline 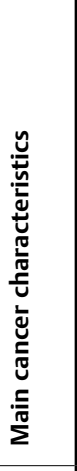 & 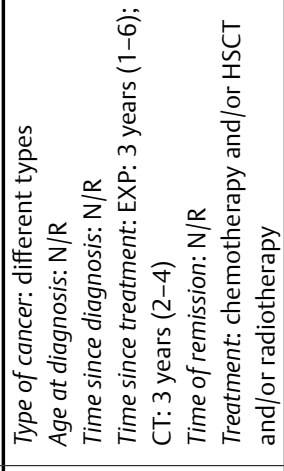 & 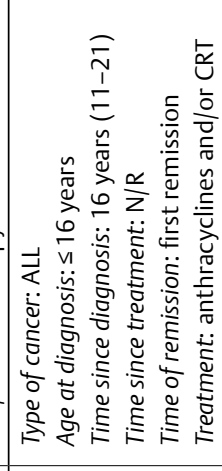 & 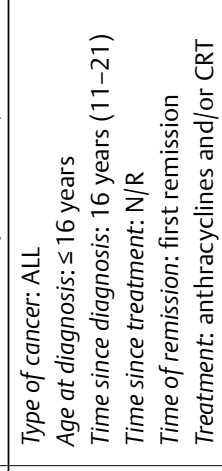 & 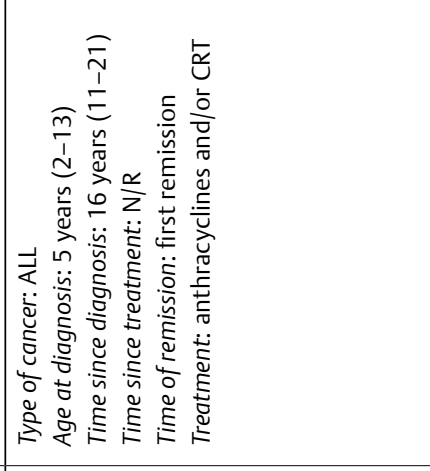 & 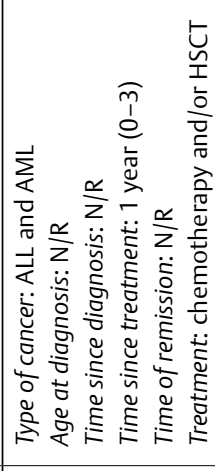 & 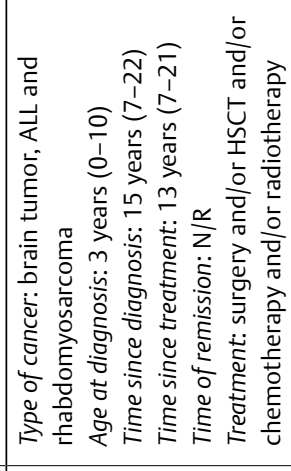 \\
\hline 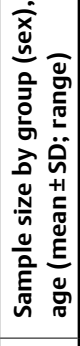 & 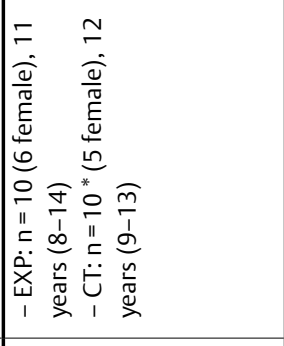 & 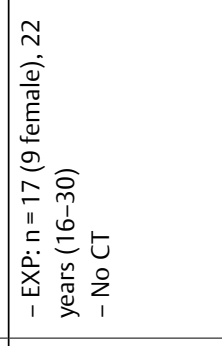 & 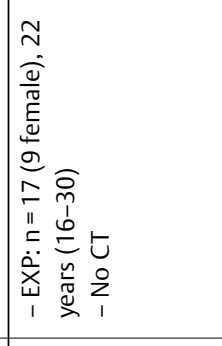 & 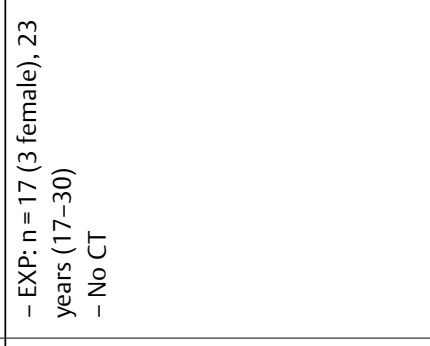 & 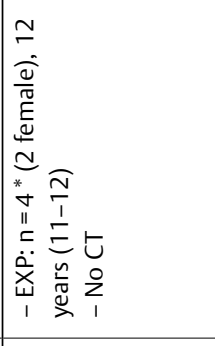 & 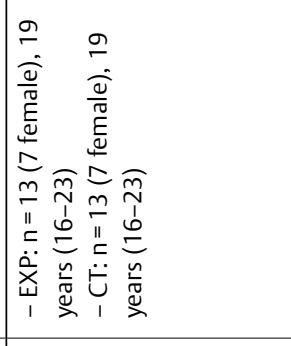 \\
\hline 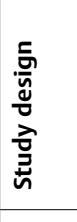 & 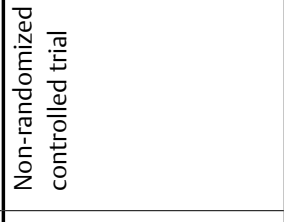 & 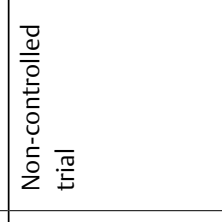 & 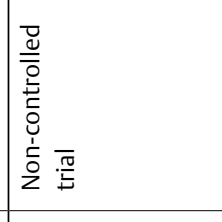 & 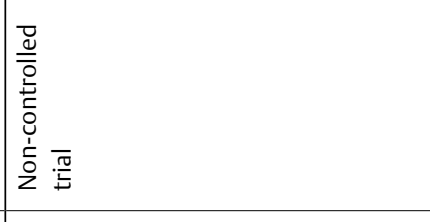 & 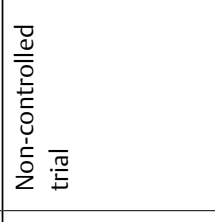 & 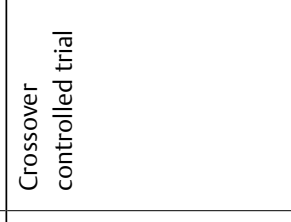 \\
\hline 剤 & 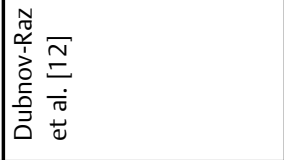 & 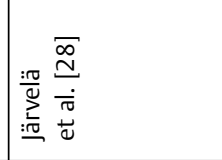 & 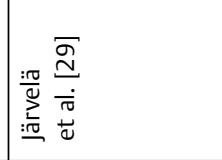 & 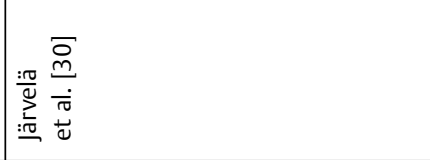 & 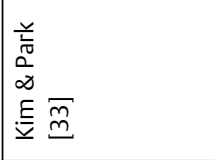 & 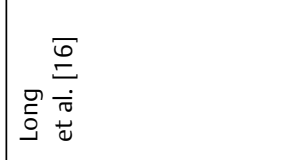 \\
\hline
\end{tabular}




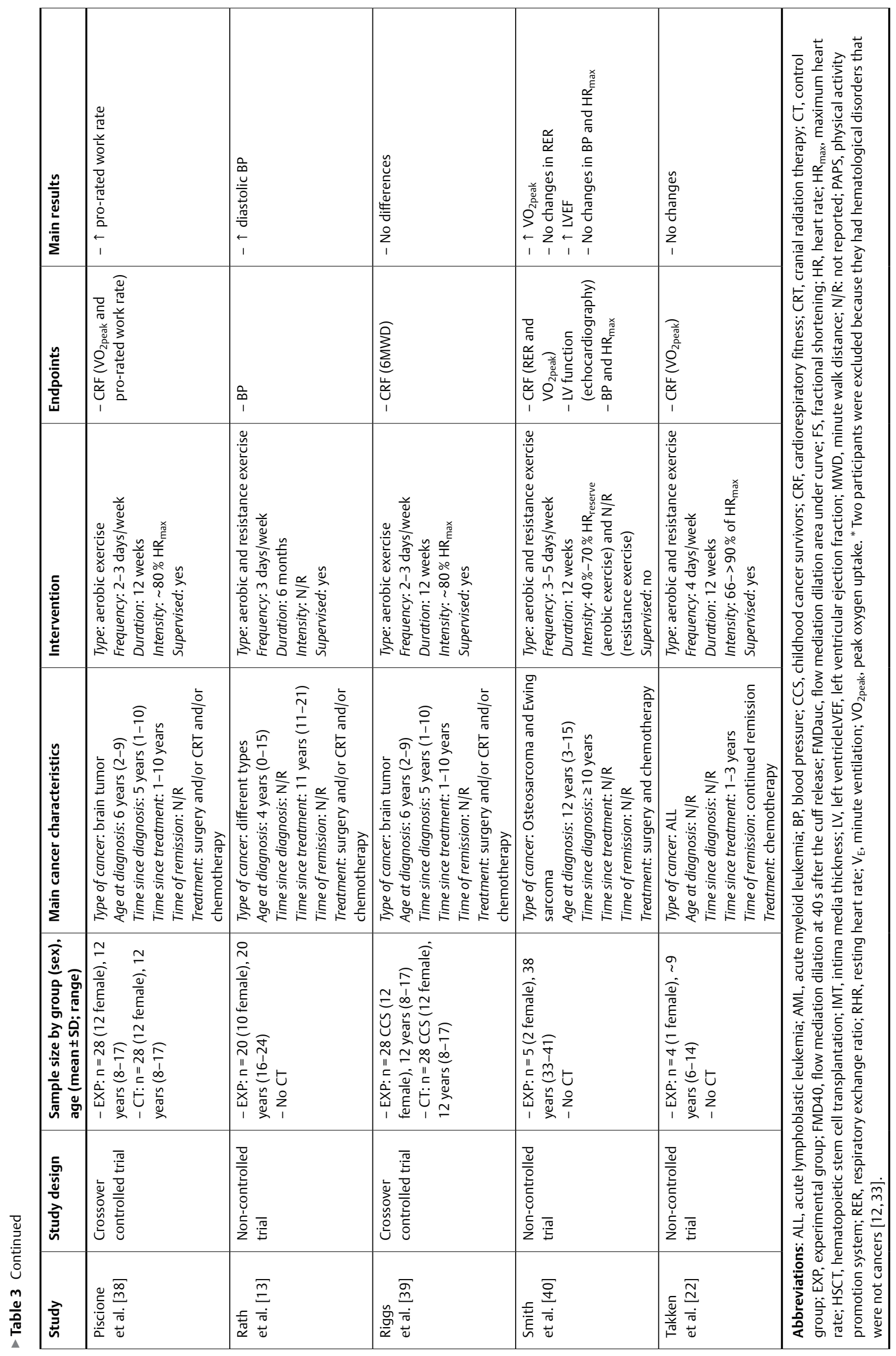


a

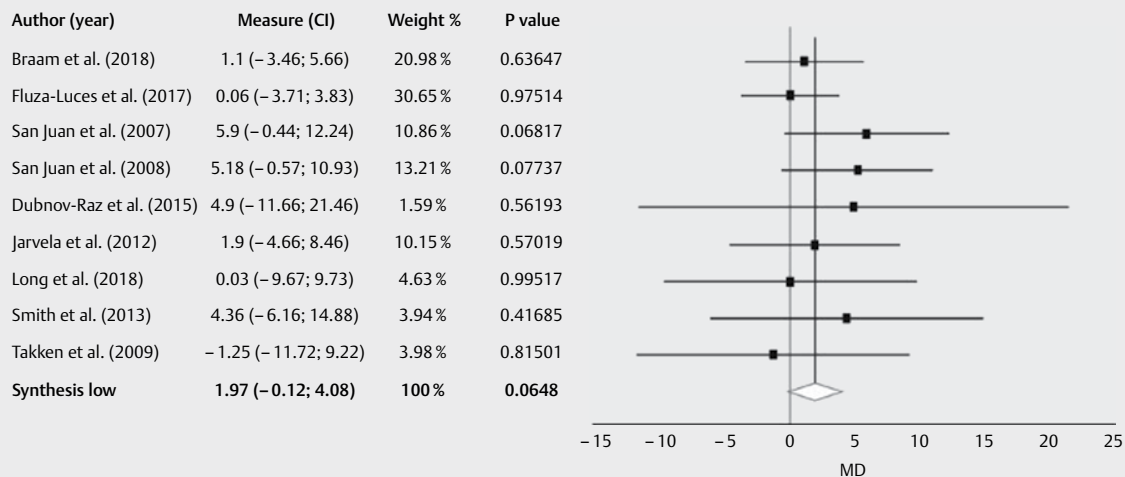

b

$\begin{array}{lccc}\text { Author (year) } & \text { Measure (Cl) } & \text { Weight \% } & \text { P value } \\ \text { Cox et al. (2017) } & 76.34(6.66 ; 146.02) & 33.74 \% & 0.03177 \\ & & & \\ \text { Esbenshade et al. (2014) } & 71.58(0.9 ; 142.26) & 33.44 \% & 0.04716 \\ & & & \\ \text { Su et al. (2018) } & 185.5(112.77 ; 258.23) & 32.82 \% & <0.00001 \\ & & & \\ \text { Synthesis low } & 110.57(38.55 ; 185.59) & 100 \% & 0.00262\end{array}$

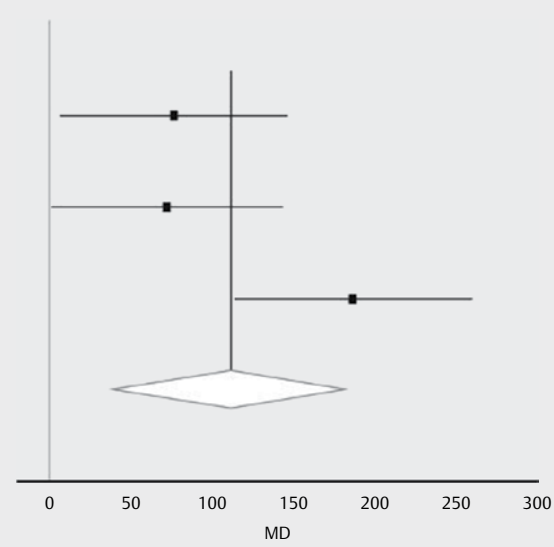

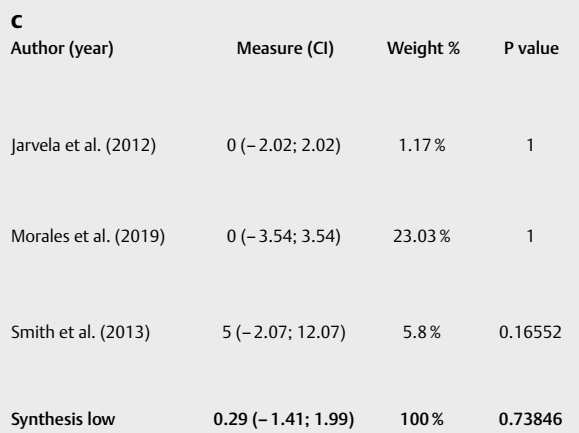

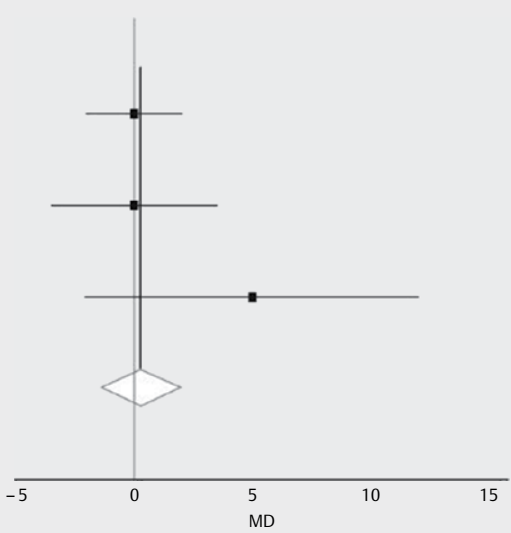

> Fig. 2 Effects (mean difference [MD] between pre-and post-intervention, expressed along with $95 \%$ confidence intervals [CI]) of exercise interventions in childhood cancer survivors on peak oxygen uptake (panel a, in $\mathrm{ml}^{\prime} \mathrm{kg}^{-1} \cdot \mathrm{min}^{-1}$ ), on the distance walked in the 6-minute walk distance test (panel $\mathbf{b}$, in meters), and on left ventricular ejection fraction (panel $\mathbf{c}$, in \%).

studies $\left(Q=6.17, \mathrm{I}^{2}=67.58 \%\right)$. No sub-analysis could be performed because the three meta-analyzed studies included CCS who were under treatment. Two studies [31,36] used the 9-minute walk distance, with one [36] reporting an increased performance after exercise and the other study [31] finding no changes. One study [33] reported an improvement in overall physical fitness - but not in CRF - as measured through the physical activity promotion system, a standardized measurement table for primary school children in
Korea that measures CRF, flexibility, muscle strength, quickness, and body mass index. No changes were found for CRF in the only study [19] that applied the Deutscher Motoriktest 6-18, a battery test that assesses endurance, strength, coordination, and flexibility. Another study [32] measured CRF using the fitnessgram, a physical fitness testing protocol that evaluates CRF, muscle strength, muscular endurance, flexibility, and body composition, finding that CRF increased at week 8 (mid-program) of the exercise interven- 
tion, but with no changes at week 16 (end of the intervention). One study [34] found an increase in CRF measured through the 20meter shuttle run test after the exercise intervention.

\section{Cardiovascular function and structure}

Four studies assessed echocardiography-determined LV systolic function [8, 28, 30, 40]. Järvelä et al. [28, 30] used the same sample, and thus we could only meta-analyze three studies. Overall, LVEF was maintained with the exercise intervention $(n=44$, $\mathrm{MD}=0.29 \%, 95 \% \mathrm{Cl}=-1.41-1.99, p=0.738$, $>$ Fig. $2 \mathrm{c}$ ) with no signs of heterogeneity $\left(Q=1.811, \mathrm{I}^{2}=0 \%\right)$ and no signs of publication bias $(p=0.296)$. Only one study [8] included a non-exercising control group, in which LVEF was significantly impaired in the control group compared with baseline at the end of the study. No sub-analyses could be performed. Although the effects on FS were analyzed in three studies, two of them $[28,30]$ shared the same sample, and thus we could not meta-analyze this endpoint. One study [8] reported that FS was preserved and reduced in the exercise and control groups, respectively, whereas in the studies by Järvelä et al. $[28,30]$, in which no control group was included, the FS was also preserved after exercise. One study [30] analyzed LV structure, finding an increase in early diastolic mitral inflow velocity and peak circumferential strain rate and diastolic strain rate after exercise.

Two studies analyzed endothelial function and structure through vascular ultrasound. Of these, one reported a decrease in intima media thickness and an increase in brachial artery flow-mediated dilation [29], whereas the other found an increase in this parameter and also in the delta diameter of the brachial artery [16].

Three studies $[16,23,40]$ analyzed the effects of exercise on different markers of heart rate. Chamorro-Viña et al. [23] found a reduction in resting heart rate after the exercise intervention in children during allogeneic hematopoietic stem cell transplant. The remaining studies found no changes in heart rate peak [40] or resting heart rate [16].

Four studies analyzed blood pressure $[13,16,28,40]$. All of them measured systolic (SBP) and diastolic blood pressure (DBP) in the sitting $[13,28,40]$ or supine $[16,28]$ position. No differences were observed for SBP $(n=59, M D=1 \mathrm{mmHg}, 95 \% \mathrm{Cl}=-3-6, p=0.605)$ or for $\mathrm{DBP}(\mathrm{n}=59, \mathrm{MD}=1 \mathrm{mmHg}, 95 \% \mathrm{Cl}=-6-8, p=0.709)$. No subanalysis could be performed because the four meta-analyzed studies included CCS who had finished treatment.

\section{Discussion}

The main finding of this systematic review and meta-analysis is that exercise interventions increased CRF in CCS, as reflected by increased performance on the 6MWD test and a trend towards improvement in $\mathrm{VO}_{2 \text { peak. }}$ Additionally, our results suggest that $\mathrm{LV}$ systolic function (i. e., LVEF) is preserved after exercise, and only three studies found any adverse event related to the exercise intervention. Therefore, exercise appears to exert a cardioprotective effect in CCS by improving - or at least attenuating the decline of - physical capacity and cardiovascular function.

Our findings show that exercise interventions increase $\mathrm{VO}_{2 \text { peak }}$ and performance on the 6MWD test (specifically, $1.97 \mathrm{ml} \cdot \mathrm{kg}^{-1} \cdot \mathrm{min}^{-1}$ and $111 \mathrm{~m}$ from baseline to post-intervention, respectively). To our knowledge, the minimal detectable change or minimal clinically important difference for this test in this patient population is unknown. However, the observed improvement in the 6MWD test was well above the minimal detectable change reported for children and adolescents with other conditions such as cystic fibrosis (i. e., 57-71 m) [41]. Cancer treatments are cardiotoxic, especially if they include anthracyclines $[4,5]$. In turn, CRF, which seems to be enhanced with exercise, is a valid predictor of overall mortality and cardiovascular risk [42]. Further, there is meta-analytical evidence showing that the $\mathrm{VO}_{2 \text { peak }}$ of survivors of childhood acute lymphoblastic leukemia is $13 \%$ lower than in healthy controls [43]. Thus, the observed exercise benefits on CRF are clinically relevant. It must be noted that a previous meta-analysis reported that aerobic exercise can have beneficial effect on CRF in CCS [9], although this meta-analysis included only studies that performed an aerobic exercise intervention.

Treatment-related cardiac death is the leading non-malignant cause of death among CCS [44]. Data from preclinical models have demonstrated the cardioprotective effects of exercise against anthracycline-induced cardiotoxicity [45-47], but the evidence in humans after cancer diagnosis remains sparse [48]. Thus, another important finding of our study is that exercise interventions seem to exert a cardioprotective effect, maintaining LVEF with respect to baseline values. In this regard, a preserved LVEF and FS were observed after a supervised in-hospital exercise intervention performed during the acute phase of treatment in CCS (neoadjuvant for solid tumors and intensive chemotherapy treatment period for leukemias), whereas both parameters were decreased during the same time period in a control group that performed no exercise [8]. Therefore, although LV function did not improve in the present meta-analysis, the fact that exercise interventions attenuated its decline is also worth noting because CCS frequently experience impaired LV function (i. e., LVEF and FS) even after treatment has ended [6]. It should be noted that in the study by Morales et al. [8], exercise-associated benefits on LV function were not maintained at 1 year of follow-up or thereafter, suggesting that exercise should be ideally maintained after treatment has ended.

Some limitations of the present systematic review include the fair methodological quality and the small sample size of most included studies (with only one out of the 27 studies including $>100$ participants). Another limitation of the present work is the heterogeneity found across studies in terms of sample characteristics (different ages, cancer types, treatments and time since diagnosis and since end of treatment), and exercise interventions (different frequency, duration, and intensity). Further research is needed to elucidate how these individual factors influence exercise benefits on CCS. Further, one of the greatest limitations we found was that some data were not available in the original studies, and although we contacted the authors to solve this problem, not all of them responded.

\section{Conclusions}

Physical exercise interventions appear to exert a cardioprotective effect in CCS by improving - or at least attenuating the decline of - physical capacity (i.e., increased performance on the 6MWD test and a trend towards an increase in $\mathrm{VO}_{2 \text { peak }}$ ) and cardiovascular function (i. e., preserved LVEF). However, more research (especially ran- 
domized controlled trials with larger sample sizes) is needed to confirm these findings.

\section{Funding}

Research by the authors is supported by the Spanish Ministry of Education, Culture and Sport [Javier S. Morales, contract \#FPU14/03435, Cecilia Rincón-Castanedo, contract\#FPU16/03956, and Alba M. Herrera-Olivares, contract \#FPU18/00215]; Universidad de Alcalá [Pedro L. Valenzuela, contract \#FPI2016]; the Spanish Ministry of Economy and Competitiveness and Fondos Feder [Alejandro Lucia, grant \#PI15/00558 and PI18/00139]; the Spanish Ministry of Economy and Competitiveness [Miguel Servet research contract (Carmen Fiuza-Luces, ref. \#CP18/00034)], 'Fundación Unoentrecienmil', 'Fundación MAPFRE' [Convocatoria Ignacio H. de Larramendi], and the National Conditioning and Strength Association (NCSA).

\section{Conflict of Interest}

The authors declare no conflicts of interest.

\section{References}

[1] Oeffinger KC, Mertens AC, Sklar CA et al. Chronic health conditions in adult survivors of childhood cancer. N Engl J Med 2006; 355: 1572-1582. doi:10.1056/NEJMsa060185

[2] Tukenova M, Guibout C, Oberlin O et al. Role of cancer treatment in long-term overall and cardiovascular mortality after childhood cancer. J Clin Oncol 2010; 28: 1308-1315. doi:10.1200/JCO.2008.20.2267

[3] Mulrooney DA, Armstrong GT, Huang $S$ et al. Cardiac outcomes in adult survivors of childhood cancer exposed to cardiotoxic therapy: A cross-sectional study. Ann Intern Med 2016; 164: 93-101. doi:10.7326/M15-0424

[4] Kremer LC, van der Pal HJ, Offringa M et al. Frequency and risk factors of subclinical cardiotoxicity after anthracycline therapy in children: A systematic review. Ann Oncol 2002; 13: 819-829

[5] Ness KK, Armenian SH, Kadan-Lottick $\mathrm{N}$ et al. Adverse effects of treatment in childhood acute lymphoblastic leukemia: General overview and implications for long-term cardiac health. Expert Rev Hematol 2011; 4: 185-197. doi:10.1586/ehm.11.8

[6] Morales JS, Valenzuela PL, Rincón-Castanedo C et al. Is health status impaired in childhood cancer survivors? A systematic review and meta-analysis. Crit Rev Oncol Hematol 2019; 142: 94-118

[7] Shaikh F, Dupuis LL, Alexander S et al. Cardioprotection and second malignant neoplasms associated with dexrazoxane in children receiving anthracycline chemotherapy: A systematic review and meta-analysis. J Natl Cancer Inst 2015; 108: djv357

[8] Morales JS, Santana-Sosa E, Santos-Lozano A et al. Inhospital exercise benefits in childhood cancer: a prospective cohort study. Scand J Med Sci Sports 2020; 30: 126-134

[9] Bourdon A, Grandy SA, Keats MR. Aerobic exercise and cardiopulmonary fitness in childhood cancer survivors treated with a cardiotoxic agent: A meta-analysis. Support Care Cancer 2018; 26: 2113-2123. doi:10.1007/s00520-018-4208-z

[10] Moher D, Liberati A, Tetzlaff J et al. Preferred reporting items for systematic reviews and meta-analyses: the PRISMA statement. J Clin Epidemiol 2009; 62: 1006-1012. doi:10.1016/j.jclinepi.2009.06.005
[11] Harriss DJ, Macsween A, Atkinson G. Standards for ethics in sport and exercise science research: 2020 update. Int J Sports Med 2019; 40: 813-817

[12] Dubnov-Raz G, Azar M, Reuveny R et al. Changes in fitness are associated with changes in body composition and bone health in children after cancer. Acta Paediatr 2015; 104: 1055-1061. doi:10.1111/apa.13052

[13] Rath SR, Long TM, Bear NL et al. Metabolic and psychological impact of a pragmatic exercise intervention program in adolescent and young adult survivors of pediatric cancer-related cerebral insult. J Adolesc Young Adult Oncol 2018; 7: 349-357. doi:10.1089/jayao.2017.0105

[14] Hozo SP, Djulbegovic B, Hozo I. Estimating the mean and variance from the median, range, and the size of a sample. BMC Med Res Methodol 2005; 5: 13. doi:10.1186/1471-2288-5-13

[15] San Juan AF, Chamorro-Viña C, Moral S et al. Benefits of intrahospital exercise training after pediatric bone marrow transplantation. Int J Sports Med 2008; 29: 439-446. doi:10.1055/s-2007-965571

[16] Long TM, Rath SR, Wallman KE et al. Exercise training improves vascular function and secondary health measures in survivors of pediatric oncology related cerebral insult. PLoS One 2018; 13: e0201449. doi:10.1371/journal.pone.0201449

[17] Verhagen AP, de Vet HC, de Bie RA et al. The Delphi list: a criteria list for quality assessment of randomized clinical trials for conducting systematic reviews developed by Delphi consensus. J Clin Epidemiol 1998; 51: 1235-1241

[18] Bax L, Yu LM, Ikeda N et al. Development and validation of MIX: comprehensive free software for meta-analysis of causal research data. BMC Med Res Methodol 2006; 6: 50. doi:10.1186/1471-2288-6-50

[19] Beulertz J, Prokop A, Rustler V et al. Effects of a 6-month, groupbased, therapeutic exercise program for childhood cancer outpatients on motor performance, level of activity, and quality of life. Pediatr Blood Cancer 2016; 63: 127-132. doi:10.1002/pbc.25640

[20] Braam KI, van Dijk-Lokkart EM, van Dongen JM et al. Cost-effectiveness of a combined physical exercise and psychosocial training intervention for children with cancer: results from the quality of life in motion study. Eur J Cancer Care (Engl) 2017; 26. doi:10.1111/ecc.12586

[21] Braam KI, van Dijk-Lokkart EM, Kaspers GJL et al. Effects of a combined physical and psychosocial training for children with cancer: A randomized controlled trial. BMC Cancer. 2018; 18: 1289. doi:10.1186/s12885-018-5181-0

[22] Takken T, van der Torre P, Zwerink M et al. Development, feasibility and efficacy of a community-based exercise training program in pediatric cancer survivors. Psychooncology 2009; 18: 440-448. doi:10.1002/pon. 1484

[23] Chamorro-Viña C, Ruiz JR, Santana-Sosa E et al. Exercise during hematopoietic stem cell transplant hospitalization in children. Med Sci Sports Exerc 2010; 42: 1045-1053. doi:10.1249/ MSS.0b013e3181c4dac1

[24] San Juan AF, Fleck SJ, Chamorro-Viña C et al. Effects of an intrahospital exercise program intervention for children with leukemia. Med Sci Sports Exerc 2007; 39: 13-21. doi:10.1249/01.mss.0000240326. 54147.fc

[25] Cox CL, Zhu L, Kaste SC et al. Modifying bone mineral density, physical function, and quality of life in children with acute lymphoblastic leukemia. Pediatr Blood Cancer 2017. doi:10.1002/pbc.26929

[26] Esbenshade AJ, Friedman DL, Smith WA et al. Feasibility and initial effectiveness of home exercise during maintenance therapy for childhood acute lymphoblastic leukemia. Pediatr Phys Ther 2014; 26 : 301-307. doi:10.1097/PEP.0000000000000053

[27] Fiuza-Luces C, Padilla JR, Soares-Miranda L et al. Exercise intervention in pediatric patients with solid tumors: The Physical Activity in Pediatric Cancer Trial. Med Sci Sports Exerc 2017; 49: 223-230. doi:10.1249/MSS.0000000000001094 
[28] Järvelä LS, Kemppainen J, Niinikoski H et al. Effects of a home-based exercise program on metabolic risk factors and fitness in long-term survivors of childhood acute lymphoblastic leukemia. Pediatr Blood Cancer 2012; 59: 155-160. doi:10.1002/pbc.24049

[29] Järvelä LS, Niinikoski H, Heinonen O] et al. Endothelial function in long-term survivors of childhood acute lymphoblastic leukemia: Effects of a home-based exercise program. Pediatr Blood Cancer 2013; 60: 1546-1551. doi:10.1002/pbc.24565

[30] Järvelä LS, Saraste M, Niinikoski H et al. Home-based exercise training improves left ventricle diastolic function in survivors of childhood all: A tissue Doppler and velocity vector imaging study. Pediatr Blood Cancer 2016; 63: 1629-1635. doi:10.1002/pbc.26051

[31] Marchese VG, Chiarello LA, Lange B]. Effects of physical therapy intervention for children with acute lymphoblastic leukemia. Pediatr Blood Cancer 2004; 42: 127-133. doi:10.1002/pbc. 10481

[32] Keats MR, Culos-Reed SN. A community-based physical activity program for adolescents with cancer (project TREK): Program feasibility and preliminary findings. J Pediatr Hematol Oncol 2008; 30: 272-280. doi:10.1097/MPH.0b013e318162c476

[33] Kim Y, Park S. Feasibility and benefits of a combined programme of exercise and play for paediatric cancer survivors: A pilot study. Eur J Cancer Care (Engl) 2019; 28: e13111

[34] Moyer-Mileur L], Ransdell L, Bruggers CS. Fitness of children with standard-risk acute lymphoblastic leukemia during maintenance therapy: Response to a home-based exercise and nutrition program. J Pediatr Hematol Oncol 2009; 31: 259-266. doi:10.1097/MPH. 0b013e3181978fd4

[35] Shore S, Shepard RJ. Immune responses to exercise in children treated for cancer. J Sports Med Phys Fitness 1999; 39: 240-243

[36] Tanir MK, Kuguoglu S. Impact of exercise on lower activity levels in children with acute lymphoblastic leukemia: A randomized controlled trial from Turkey. Rehabil Nurs 2013; 38: 48-59. doi:10.1002/rnj.58

[37] Su HL, Wu LM, Chiou SS et al. Assessment of the effects of walking as an exercise intervention for children and adolescents with cancer: A feasibility study. Eur J Oncol Nurs 2018; 37: 29-34. doi:10.1016/j. ejon.2018.10.006

[38] Piscione PJ, Bouffet E, Timmons B et al. Exercise training improves physical function and fitness in long-term paediatric brain tumour survivors treated with cranial irradiation. Eur J Cancer 2017; 80: 63-72. doi:10.1016/j.ejca.2017.04.020
[39] Riggs L, Piscione J, Laughlin S et al. Exercise training for neural recovery in a restricted sample of pediatric brain tumor survivors: A controlled clinical trial with crossover of training versus no training. Neuro Oncol 2017; 19: 440-450. doi:10.1093/neuonc/now177

[40] Smith WA, Ness KK, Joshi V et al. Exercise training in childhood cancer survivors with subclinical cardiomyopathy who were treated with anthracyclines. Pediatr Blood Cancer 2013. doi:10.1002/pbc.24850

[41] López-de-Uralde-Villanueva I, Sarría Visa T, Moscardó Marichalar P et al. Minimal detectable change in six-minute walk test in children and adolescents with cystic fibrosis. Disabil Rehabil 2019; 19: 1-6. doi: 10.1080/09638288.2019.1663947

[42] Kodama S, Saito K, Tanaka S et al. Cardiorespiratory fitness as a quantitative predictor of all-cause mortality and cardiovascular events in healthy men and women: A meta-analysis. JAMA 2009; 301: 2024-2035. doi:10.1001/jama.2009.681

[43] van Brussel M, Takken T, Lucia A et al. Is physical fitness decreased in survivors of childhood leukemia? A systematic review. Leukemia 2005; 19: 13-17. doi:10.1038/sj.leu.2403547

[44] Mertens AC, Liu Q, Neglia JP et al. Cause-specific late mortality among 5-year survivors of childhood cancer: The Childhood Cancer Survivor Study. J Natl Cancer Inst 2008; 100: 1368-1379. doi:10.1093/jnci/ $\operatorname{djn} 310$

[45] Ahmadian M, Dabidi Roshan V. Modulatory effect of aerobic exercise training on doxorubicin-induced cardiotoxicity in rats with different ages. Cardiovasc Toxicol 2018; 18: 33-42. doi:10.1007/s12012-0179411-5

[46] Wang F, Iskra B, Kleinerman E et al. Aerobic exercise during early murine doxorubicin exposure mitigates cardiac toxicity. J Pediatr Hematol Oncol 2018; 40: 208-215. doi:10.1097/MPH. 0000000000001112

[47] Parry TL, Hayward R. Exercise training does not affect anthracycline antitumor efficacy while attenuating cardiac dysfunction. Am J Physiol Regul Integr Comp Physiol 2015; 309: R675-R683. doi:10.1152/ ajpregu.00185.2015

[48] Scott JM, Nilsen TS, Gupta D et al. Exercise therapy and cardiovascular toxicity in cancer. Circulation 2018; 137: 1176-1191. doi:10.1161/ CIRCULATIONAHA.117.024671 